\title{
The Role of Monocyte/Macrophage and CXCR3 in Differentiation between Recurrent Hepatitis $C$ and Acute Cellular Rejection Postliver Transplantation
}

\author{
Asmaa Ibrahim Gomaa, ${ }^{1}$ Nermine Ahmed Ehsan $\left(\mathbb{D},{ }^{2}\right.$ Ahmed A. Elrefaei, \\ Mervat Mohamed Sultan, ${ }^{2}$ and Maha Mohamed Elsabaawy ${ }^{1}$ \\ ${ }^{1}$ Department of Hepatology and Gastroenterology, National Liver Institute, Menoufia University, Shebin El Koum, Egypt \\ ${ }^{2}$ Department of Pathology, National Liver Institute, Menoufia University, Shebin El Koum, Egypt \\ Correspondence should be addressed to Nermine Ahmed Ehsan; nermine_ehsan@yahoo.com
}

Received 6 January 2018; Revised 8 March 2018; Accepted 1 April 2018; Published 30 April 2018

Academic Editor: Marcella Reale

Copyright (C) 2018 Asmaa Ibrahim Gomaa et al. This is an open access article distributed under the Creative Commons Attribution License, which permits unrestricted use, distribution, and reproduction in any medium, provided the original work is properly cited.

\begin{abstract}
Objective. Liver transplantation (LT) is the recommended treatment for patients with advanced liver disease and cirrhosis in all guidelines, mostly as a complication of HCV. The distinction between reinfection of the graft with $\mathrm{HCV}$ and acute cellular rejection (ACR) is essential because they are managed differently. Hepatic macrophages, which can either arise from circulating blood-derived monocytes (BDM) or from resident tissue Kupffer cells, are central in the pathogenesis of chronic liver injury. The aim of this work was to evaluate whether the origin of macrophages and the immune mediator CXCR3 could help in differentiating between acute recurrent $\mathrm{HCV}$ and ACR after liver transplantation. Methods. Twenty-nine cases of recurrent hepatitis C and 26 cases of ACR were included in this study. The expression of CD 68 (macrophage marker), CD11b (BDM marker), and CxCR3 in the postliver transplant biopsy using immunohistochemistry was determined. Results. CD11b expression highlighting macrophages of BDM origin was in favor of recurrent hepatitis $\mathrm{C}(P<0.001)$ than in ACR $(P=0.44)$, while CXCR3 expression by hepatocytes was in favor of ACR $(P=0.001)$. Conclusion. Macrophage infiltrating liver tissue post LT can distinguish between ACR by upregulation of CXCR3 and recurrent hepatitis C by predominant CD11b.
\end{abstract}

\section{Introduction}

Chronic hepatitis $\mathrm{C}$ virus infection (HCV) is a major cause of end-stage liver disease that has been increasingly the important indication for liver transplantation (LT) globally. HCV reinfection of the graft occurs almost universally, leading to graft injury in the majority of patients and cirrhosis in 8 $44 \%$ in 5-7 years after reinfection [1]. The viral load may be influenced by corticosteroid intake [2], and the histologic features of recurrent hepatitis $\mathrm{C}$ may be modified by immunosuppressive therapy, which harden its differentiation from acute cellular rejection (ACR) [3]. Allograft failure is the most common cause of death and retransplantation among those recipients [4-7].
ACR is encountered in $18 \%-30 \%$ of transplanted patients leading to allograft failure $[8,9]$. The differential diagnosis between recurrent HCV and ACR is often difficult due to the same clinical picture, and laboratory abnormalities detected in both diseases, and even similar histological features [10]. Moreover, low interobserver and intraobserver agreement rates were found among experienced liver transplant pathologists for the histopathologic differentiation of recurrent hepatitis C from ACR [3].

IFN-free direct-acting antiviral agents (DAAs) have improved tolerability and can potentially be used in posttransplant setting, which should result in better outcomes [11]. However, incorrect diagnosis may be detrimental, as failure to increase immunosuppression in patients with 
ACR may lead to acceleration of rejection, and inappropriate treatment of suspected acute rejection with high-dose pulse steroid therapy in misdiagnosed recurrent HCV can lead to aggravation of the disease, graft loss, and poor survival; hence, accurate diagnosis remains a critical issue $[8,12]$.

Macrophages hold a fundamental role in regulating inflammatory processes [13]. In particular, hepatic macrophages have the main role in the pathogenesis of acute and chronic liver injury through a wide range of different functions in the liver. The liver has about $80 \%$ of all body macrophages as local resident self-renewing macrophages, termed Kupffer cells. Blood monocytes can infiltrate into the liver; however, under steady-state conditions, blood monocytederived macrophages (BDM) do not contribute to the pool of local resident macrophages in the liver [14]. Previous work demonstrated that a reduction in number and function of circulating monocytes are strongly correlated with activation of systemic anti-inflammatory responses [15]. Macrophages in the liver can be distinguished based on their origin and certain marker expression. CD68 (cluster of differentiation 68 ) is a glycoprotein which binds low-density lipoprotein and is expressed on monocytes/macrophages [16]. Yang et al. [17] demonstrated that early activation of macrophages as a result of graft injury might play an important role in the accelerated ACR.

However, patients with HCV infection may have a significant increase in CD68+ expression in their portal tracts compared with normal tissue [18].

CXCL10 is well known in hepatitis C as a hepatocytederived chemotactic ligand and initiator of inflammatory cascades via its cognate receptor C-X-C motif receptor 3 (CXCR3). It is widely expressed on multiple cells of the innate immune system, including hepatic Kupffer cells, dendritic cells, natural killer (NK) cell, and neutrophils. Hence, these entire different innate immune cells are potential targets for CXCL10-mediated chemotaxis. [19]. Treatment with a CXCL11-neutralizing antibody reduced the number of CXCR3 + cells in the skin allograft and prolonged graft survival [20].

Till now, no precise marker for diagnosing ACR or recurrent $\mathrm{HCV}$ is currently available. This study aimed to determine the role of CD68, CD 11b, and CXCR3, as markers of resident Kupffer cells and BDM, in the differentiation between HCV reinfection and ACR in the postliver transplant setting using immunohistochemistry.

\section{Methods}

2.1. Specimens' Selection. This retrospective study was conducted on liver biopsies from 55 patients who had living donor liver transplantation (LDLT) for chronic HCV complications, whether cirrhosis and/or HCC, and who had developed elevated liver enzymes 6 months following transplantation. Twenty-nine patients developed recurrent $\mathrm{HCV}$, and 26 patients were diagnosed as ACR based on histopathological examination. Laboratory investigation and histological criteria established the diagnosis which was confirmed by good response to treatment. Serological and clinical data were collected from the patients' files.
The study was approved by the National Liver Institute Institutional Review Board.

Paraffin-embedded blocks of those liver biopsies were retrieved from the archive of the Pathology Department, National Liver Institute, Menoufia University, in the period between 2015 and 2017. Baseline characteristics including donor and recipient age and gender, pretransplantation HCC status, liver function tests, HCV-RNA level, and MELD score were determined.

2.2. Histopathological Evaluation. Serial liver sections in four micrometer thickness were cut from each paraffin-embedded block, for hematoxylin and eosin (H\&E) staining and immunostaining. H\&E staining was used for evaluation of histopathological changes including determination of the following parameters:

(i) Extent of infiltrate and the degree of portal inflammation identified by mononuclear infiltration of portal tracts

(ii) Presence of interface hepatitis, spotty necrosis, confluent necrosis, steatosis, and cholestasis

(iii) Presence or absence of fibrosis

(iv) Presence of bile duct injury, venous endothelial injury, hepatic artery injury, or perivenular necrosis

(v) The nature and number of portal tract infiltrate: plasma cells, eosinophils, neutrophils, macrophages, and immunoblast cells

2.3. Immunohistochemistry. Immunohistochemistry was carried out for all tissues mentioned in the study. After deparaffinization and rehydration, hydrogen peroxide was applied to block nonspecific background staining. Heatinduced antigen retrieval was performed using citrate buffer solution low $\mathrm{pH}$ ( $\mathrm{pH}$ 6) for CD11b and CX3CR1 antibodies and high $\mathrm{pH}(\mathrm{pH} 9)$ for CD68. They are anti-human antibodies that arose in animals and recognize CD68 antigens on human macrophages [21]. Antigen retrieval solution was performed in a vegetable steamer for 20 minutes at $97^{\circ} \mathrm{C}$ followed by incubation for an additional 20 minutes in the warm buffer. All antibodies were incubated overnight at $4^{\circ} \mathrm{C}$. Sections were incubated with a monoclonal mouse CD68 (clone KP1,0, DAKO A/S, Glostrup, Denmark, dilution 1:50), a rabbit polyclonal primary anti CD11 b (Novus Biologicals, Littleton, CO, USA, dilution 1:50), or a $1: 200$ dilution of a rabbit polyclonal primary anti-CX3CR1 antibody (Novus Biologicals, Littleton, CO, USA). Detection of the immunostaining was carried out utilizing the EnVisionTM FLEX/HRP detection system (DAKO A/S, Glostrup, Denmark) with the 3-diaminobenzidine (DAKO) as chromogen. After counterstaining with Mayer's hematoxylin, the slides were independently assessed by two pathologists for detection of each antibody.

Human lymph node (stains sinusoids) was positive tissue control for CD68; benign prostatic hyperplasia was positive tissue control for CD11 and human heart tissue for CX3CR1. 
Negative tissue controls were included in the protocol of staining by omitting the primary antibodies.

2.4. Interpretation of CD68, CD11b, and CXCR3. The immunoreactivity for CD68 was identified as membranous brownish discoloration of macrophages. The positive cells were quantified in three portal tracts and adjacent hepatic parenchyma per case (liver core). The positive cases were further divided according to the median number of CD68 positive cells into high expression $(>40 \%)$ and low expression $(<40 \%)$. The immunoreactivity for CD11b was assigned when cytoplasmic brownish discoloration was seen in mononuclear inflammatory cells. The positive cells were quantified in three portal tracts, interface, and adjacent hepatic parenchyma. The percentage of positivity was evaluated and expressed as range, mean, and median. The cases were divided into low expression when up to $30 \%$ of hepatocytes were positive and high expression when $>30 \%$ of hepatocytes showed immunoreactivity. The immunoreactivity for CXCR3 was identified as cytoplasmic brownish discoloration of mononuclear inflammatory cells. The positive cells were quantified in three portal tracts and adjacent parenchyma per case (liver core). The positive cases were further divided according to the median number of CXCR3 positive cells into high expression $(>20 \%)$ and low expression $(<20 \%)$.

2.5. Statistical Analysis. Qualitative data was expressed in number and percentages, and quantitative data was expressed as mean and standard deviation. Fisher exact and chi-square tests were used to study the association between two qualitative variables. $t$-test was used for comparison between two quantitative variables. A $P$ value of $<0.05$ was considered statistically significant.

\section{Results}

The baseline clinical, laboratory data of the studied patients are presented in Table 1. No significant difference was observed between the two groups regarding recipient age, gender, MELD score, and presence of HCC before transplantation $(P=0.11,0.87,0.57$, and 0.54 , resp.).

Histopathological features of recurrent chronic hepatitis $\mathrm{C}(\mathrm{CHC})$ and ACR are demonstrated in Table 2.

3.1. CD68 Expression. CD68 was detected in the inflammatory infiltrate in all cases of recurrent hepatitis C and ACR and was localized in portal tracts and adjacent parenchyma. The number of macrophages identified by CD68 immunostaining ranged from 10 to 60 in both groups. In recurrent hepatitis $C$, the mean $\pm S D$ was $32.3 \pm 17.5$ and a median of 30.0 , while in cases of ACR, the mean \pm SD was $37.7 \pm 12.4$ and a median of 40.0 without detectable significant difference between the two groups $(P=0.21)$ as shown in Table 3 . Twelve cases (46.2\%) of recurrent hepatitis $C$ showed high expression of CD68 in comparison to 17 patients (65.4\%) in ACR.

3.2. CD11b Expression. CD11b expression was detected among the mononuclear inflammatory infiltrate in portal areas, interface, or hepatic parenchyma. In recurrent hepatitis
TABLE 1: Demographic and laboratory characteristics of patients with recurrent chronic hepatitis $\mathrm{C}(\mathrm{CHC})$ and acute cellular rejection (ACR).

\begin{tabular}{lcc}
\hline Parameters & $\begin{array}{c}\text { Recurrent CHC } \\
(n=29) \%\end{array}$ & $\begin{array}{c}\text { ACR } \\
(n=26) \%\end{array}$ \\
\hline Recipient age (years) & $47.9 \pm 5.7$ & $42.2 \pm 13.4$ \\
Gender of recipient & $24(82.7) / 5(17.3)$ & $26(100) / 0(0)$ \\
(M/F) $n, \%$ & $8(27.6)$ & $7(26.9)$ \\
Pretreatment HCC $n, \%$ & $15.8 \pm 2.6$ & $15.4 \pm 2.0$ \\
MELD score & $4.11 \pm 5.0$ & $5.1 \pm 2.9$ \\
Total bilirubin (mg/dL) & $127.1 \pm 65.1$ & $198.8 \pm 164.1$ \\
AST (IU/L) & $165.9 \pm 111.9$ & $269.7 \pm 257.6$ \\
ALT (IU/L) & $721.7 \pm 1041.4$ & $1054.3 \pm 1232.2$ \\
GGT (IU/L) & $371.6 \pm 211.3$ & $400.9 \pm 267$ \\
ALP (IU/L) & $4.22 \pm 0.33$ & $3.97 \pm 0.49$ \\
Serum albumin (gm/dL) &
\end{tabular}

AST: aspartate aminotransferase; ALT: alanine aminotransferase; GGT: gamma glutamyl transferase; ALP: alkaline phosphatase.

$\mathrm{C}$, the number of macrophages identified by CD11b immunostaining ranged from 10 to 60 ; the mean $\pm \mathrm{SD}$ was $26.5 \pm 17.2$ and the median was 30.0 , while in ACR, ranging from 10 to 50 , the mean \pm SD was $17.3 \pm 12.5$ and a median of 10.0 , with a significant difference detected between the two groups $(P=0.03)$. High expression of CD11b $(\geq 30 \%)$ was found in $14(53.8 \%)$ and 5 cases (19.2\%) of recurrent HCV and ACR, respectively $(P=0.01)$.

3.3. CXCR3 Expression. CXCR3 expression was shown among the mononuclear inflammatory infiltrate in portal tracts, interface, or hepatic parenchyma. A significant difference between the two groups was detected $(P<0.001)$. The number of macrophages identified by CXCR3 immunostaining in recurrent hepatitis $\mathrm{C}$ ranged from 0 to 30; the mean $\pm \mathrm{SD}$ was $6.2 \pm 8.5$ and the median was 1.0. While in ACR, it ranged from 0 to 40 ; the mean \pm SD was $20.4 \pm 10.4$ and the median was 20.0. High expression of CXCR3 $(\geq 20 \%)$ was found in $2(7.7 \%)$ versus $18(69.2 \%)$ cases of recurrent HCV and ACR, respectively $(P<0.001)$. Figure 1 demonstrated immunohistochemical staining of CD68, CD11b, and CXCR3 in recurrent hepatitis C (Figures 1(a), 1(c), and 1(e)) and acute cellular rejection (Figures 1(b), 1(d), and $1(\mathrm{f}))$.

Subgroup analysis was performed according to presence or absence of HCC and revealed the same trend, although CD11b expression was not statistically significant between both groups (Tables 4 and 5).

Histological criteria established the diagnosis which was confirmed by good response to treatment in $94 \%$ of cases. Three cases were confusing and reassessment of the biopsy or even rebiopsy was mandatory. They are misdiagnosed as recurrent HCV posttransplantation, however, during follow-up, one patient was discovered to have lymphoproliferative malignancy and improved upon receiving systemic chemotherapy. The other two patients were found to have hyperacute and chronic rejection that unfortunately did not respond to increasing dose of immunosuppressive drugs 
TABLE 2: Histopathological characteristics of patients with recurrent chronic hepatitis $\mathrm{C}$ (CHC) and acute cellular rejection (ACR).

\begin{tabular}{lcc}
\hline Parameters & Recurrent CHC $(n=29) \%$ & ACR $(n=26) \%$ \\
\hline Extent of infiltrate $n, \%$ & & $10(38.5)$ \\
$\quad$ I & $7(24.13)$ & $14(53.8)$ \\
$\quad$ II & $17(58.62)$ & $2(7.7)$ \\
$\quad$ III & $5(17.24)$ & $1(3.8) / 25(96.2)$ \\
Spotty necrosis (absent/present) $n, \%$ & $0(0) / 29(100)$ & $23(88.5) / 3(11.5)$ \\
Confluent necrosis (absent/present) $n, \%$ & $5(17.24) / 24(82.76)$ & $8(30.8) / 18(69.2)$ \\
Perivenular necrosis (absent/present) $n, \%$ & $20(68.96) / 9(31.04)$ & $18(69.2) / 8(30.8)$ \\
Fibrosis (absent/present) $n, \%$ & $9(31.04) / 20(68.96)$ & $17(65.4) / 9(34.6)$ \\
Cholestasis (absent/present) $n, \%$ & $23(79.3) / 6(20.7)$ & $22(84.6) / 4(15.4)$ \\
Steatosis (absent/present) $n, \%$ & $4(13.8) / 25(86.2)$ & $3(11.5) / 23(88.5)$ \\
Bile duct injury (absent/present) $n, \%$ & $16(55.2) / 13(44.8)$ & $4(15.4) / 22(84.6)$ \\
Vascular injury (absent/present) $n, \%$ & $28(96.5) / 1(3.5)$ & \\
\hline
\end{tabular}

TABLE 3: Comparison between recurrent chronic hepatitis $\mathrm{C}(\mathrm{CHC})$ and acute cellular rejection (ACR) regarding the studied markers (CD68, CD11b, and CXCR3).

\begin{tabular}{lcccc}
\hline Marker & Recurrent CHC & ACR & $t$-test & $P$ value \\
\hline CD68 & & & & 0.21 \\
Mean \pm SD & $32.3 \pm 17.5$ & $37.7 \pm 12.4$ & -1.27 & \\
Median & 30.0 & 40.0 & & 0.03 \\
CD11b & & & & \\
Mean \pm SD & $26.5 \pm 17.2$ & $17.3 \pm 12.5$ & 2.21 & \\
Median & 30.0 & 10.0 & & 0.001 \\
CXCR3 & & & & \\
Mean \pm SD & $6.2 \pm 8.5$ & $20.4 \pm 10.4$ & -5.40 & \\
Median & 10.0 & 20.0 & & \\
\hline
\end{tabular}

and died. Another two patients diagnosed with ACR also died due to sepsis.

\section{Discussion}

Recurrent hepatitis $\mathrm{C}$ is characterized by the presence of lobular inflammation, apoptotic bodies, spotty necrosis, and lobular disarray, with portal lymphocyte predominance, while, in acute cellular rejection mixed portal/periportal inflammation composed of lymphocytes, plasma cells, and eosinophils, lymphocytic cholangitis and endothelialitis were observed. However, the histological diagnosis of HCV infection in the transplant setting may be altered, putting in mind that detectable serum HCV ribonucleic acid (RNA) after LT, even at a high level, does not necessarily indicate the presence of histologic recurrent hepatitis C [3]. Immunosuppression may alter the histological appearance of viral hepatitis posttransplant especially during the first few months; hence, classic histopathologic features of hepatitis $\mathrm{C}$ may be absent or modified. Moreover, ACR, ischemic injury, biliary obstruction, cytomegalovirus infection, or drug toxicity may superimpose chronic hepatitis [3]. Therefore, it is a challenge for the hepatopathologist to differentiate these overlapping microscopic features in some cases.
The present study aimed to determine the usefulness of macrophages' origin in the differential diagnosis of acute rejection and recurrent $\mathrm{HCV}$ after LT.

Macrophages have an important role in both recurrent hepatitis C and ACR posttransplantation. About $80 \%$ of all body macrophages reside in the liver and are furthermore patrolled by blood monocytes [22]. The circulating blood monocytes can principally infiltrate the liver and give rise to monocyte-derived macrophages, but this is characteristic to liver injury [23]. Liver macrophages have a wide range of functional heterogeneity; they may be pathogenic or even beneficial and they have been classified either into "proinflammatory" M1 or "immunoregulatory” M2 macrophages. Macrophages play a key role in acute and chronic liver inflammation and regression of liver disease. Upon injury to the liver, macrophages often perform immediate multiple functions including cytokine and chemokine secretion, leukocyte adhesion, phagocytosis, angiogenesis control, and extracellular matrix remodelling [24]. Although Kupffer cells can protect the transplanted liver, rejection of allografts was found also promoted by macrophages due to their antigenpresenting and cytokine-releasing function [24]. In cases of ACR, selective targeting and destruction of donor parenchymal cells occur through complement activation and the resultant membrane attack complex is responsible for lysis of the donor cells. Another pathway for the destruction of donor parenchymal cells in ACR is via antibody-dependent cellular cytotoxicity, involving other immune cell mediators, such as NK cells, macrophages (CD68-positive cells), and neutrophils [25].

CD68 has been proposed as an indicator for Kupffer cells [26] and is used to distinguish Kupffer cells from monocytederived macrophages $[23,27]$. However, no single marker is currently able to definitely discriminate these populations. CD68 is a specific marker for the various cells of the macrophage lineage, including monocytes, Kupffer cells, histiocytes, giant cells, and osteoclasts. In this study, CD68 was expressed in all cases of recurrent hepatitis C and ACR, indicating the presence of macrophage infiltration of portal tracts in both groups. There was no significant difference in the number of macrophages highlighted by CD68 between cases 


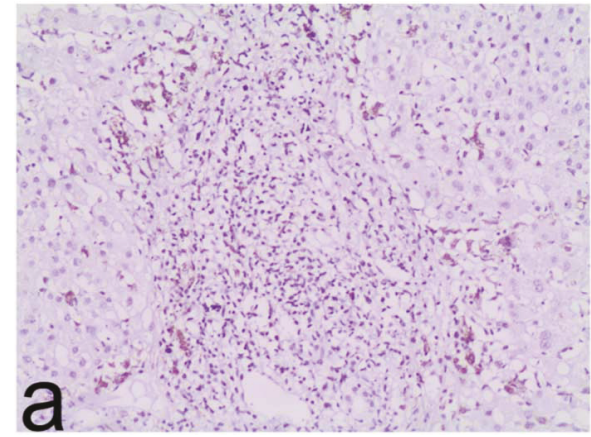

(a)

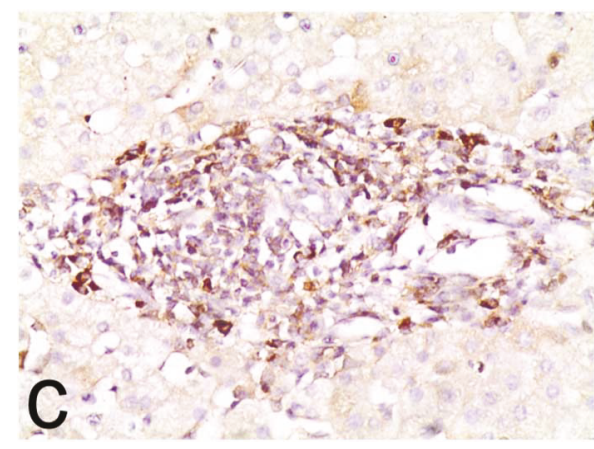

(c)

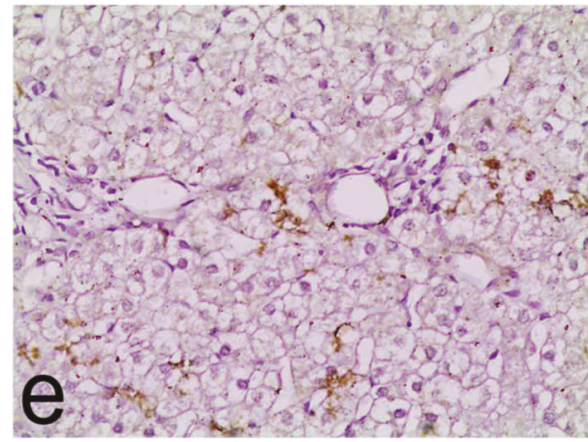

(e)

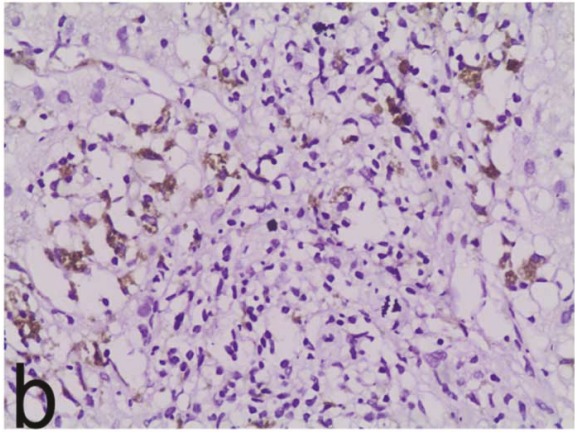

(b)

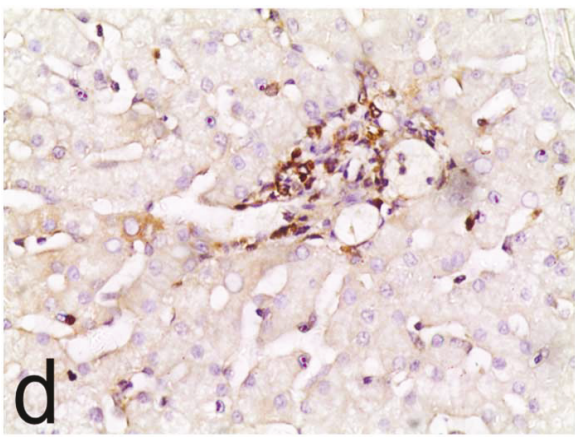

(d)

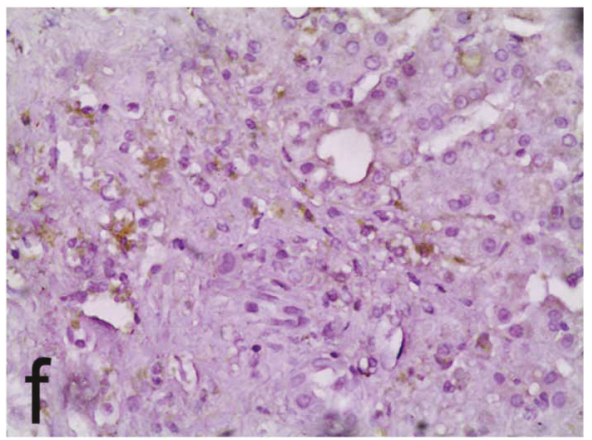

(f)

FIGURE 1: Immunohistochemistry of liver tissue postliver transplantation in recurrent hepatitis $\mathrm{C}(\mathrm{a}, \mathrm{c}$, and e) and acute cellular rejection (b, d, and $\mathrm{f}$ ) showing immunoreaction for CD68 ( $\mathrm{a}$ and b), CD11b ( $\mathrm{c}$ and d), and CXCR3 (e and f). Original magnification $\times 200$.

TABLE 4: Comparison between recurrent chronic hepatitis $\mathrm{C}(\mathrm{CHC})$ and acute cellular rejection (ACR) regarding the studied markers (CD68, CD11b, and CXCR3) in absence of HCC.

\begin{tabular}{lcccc}
\hline $\begin{array}{l}\text { Marker } \\
(\text { mean } \pm \text { SD) }\end{array}$ & Recurrent CHC & ACR & $t$-test & $P$ value \\
\hline CD68 & $33.5 \pm 17.3$ & $36.7 \pm 11.7$ & -0.59 & 0.56 \\
CD11b & $26.5 \pm 15.8$ & $18.6 \pm 15.5$ & 1.40 & 0.17 \\
CXCR3 & $7.6 \pm 9.7$ & $18.0 \pm 10.8$ & -2.85 & 0.008 \\
\hline
\end{tabular}

of recurrent hepatitis $\mathrm{C}$ or $\mathrm{ACR}(P=0.21)$. In accordance with this finding, CD68+ macrophages were found in the infiltrate of hepatic lobules in acute liver allograft rejection and the number of infiltrating cells correlated with the severity of the ACR in a previous report [28]. Also, the number of CD14+CD68+ Kupffer cells is increased in patients with viral
TABLE 5: Comparison between recurrent chronic hepatitis $\mathrm{C}$ (CHC) and acute cellular rejection (ACR) regarding the studied markers (CD68, CD11b, and CXCR3) in presence of HCC.

\begin{tabular}{lcccc}
\hline $\begin{array}{l}\text { Marker } \\
(\text { mean } \pm \text { SD })\end{array}$ & Recurrent CHC & ACR & $t$-test & $P$ value \\
\hline CD68 & $30.0 \pm 18.7$ & $38.6 \pm 15.7$ & -0.97 & 0.35 \\
CD11b & $26.6 \pm 20.6$ & $14.3 \pm 5.3$ & 1.53 & 0.15 \\
CXCR3 & $3.3 \pm 5.0$ & $24.3 \pm 11.3$ & -4.99 & $<0.001$ \\
\hline
\end{tabular}

hepatitis in another study [29]. In addition, CD68-positive monocytes were the main inflammatory cell-infiltrating renal graft in cases of ACR [30].

Monocyte-derived (freshly infiltrating) macrophages are

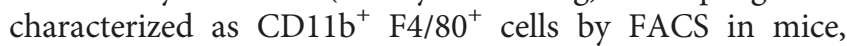
whereas matured monocyte-derived and resident Kupffer 
cells are $\mathrm{CD} 11 \mathrm{~b}^{\mathrm{lo}} \mathrm{F} 4 / 80^{\mathrm{hi}}[31]$. The number of $\mathrm{CD} 11 \mathrm{~b}(+), \mathrm{F} 4 /$ $80(+)$, CD11c(-), and CD206(+) (M2) macrophages in the liver of HCV transgenic mice was notably increased compared to control mice. These M2 macrophages in the liver produced elevated levels of IL- 6 and TNF- $\alpha$. These results suggested that inflammatory cytokines produced by M2like macrophages contribute to the induction of chronic liver inflammation in HCV transgenic mice [32]. In agreement with these results, the present study demonstrated that the $\mathrm{CD} 11 \mathrm{~b}$ expression was in favor of recurrent hepatitis $\mathrm{C}$ compared with ACR $(P=0.03)$.

A massive necrosis of hepatocytes can provoke a strong inflammatory immune response within the liver [33] leading to secretion of diverse proinflammatory chemokines and cytokines, including interferon (IFN)- $\gamma$ by liver-resident and -infiltrating immune cells [34] which perpetuate liver cell damage. IFN $-\gamma$ strongly activates the transcription of the chemokines CXCL9, CXCL10, and CXCL11 [35, 36]. CXCR3, one of the peripheral blood monocyte surface markers, is the receptor for CXCL9, CXCL10, and CXCL11 chemokines which is expressed on various cell subpopulations within the liver, including liver endothelial cells, stellate cells, T cells, NK cells, and NKT cells $[37,38]$. The interaction between these three chemokines and their receptor mediates the recruitment of T, NK, and NKT cells into the liver and their attachment to endothelial cells [39-41].

Our results clearly show great differences in CXCR3 expression between both groups. CXCR3 expression was significantly higher in ACR than recurrent HCV.

In conclusion, CD68 was expressed in both recurrent $\mathrm{HCV}$ infection and ACR. A significantly stronger CD11b deposits in liver biopsies of patients' suffering from recurrent HCV was detected. On the other hand, CXCR3 was a marker and plays a considerable role in acute rejection following liver transplantation suggesting the involvement of humoral mechanisms in ACR. Using immunohistochemistry beside clinical, laboratory, and histopathological criteria in discrimination between recurrent $\mathrm{HCV}$ and ACR may improve the diagnostic ability, morbidity, and mortality of these patients.

\section{Conflicts of Interest}

The authors declare that they have no conflicts of interest.

\section{References}

[1] N. A. Terrault, "Treatment of recurrent hepatitis C in liver transplant recipients," Clinical Gastroenterology and Hepatology, vol. 3, no. 10, Supplement 2, pp. S125-S131, 2005.

[2] R. B. Guerrero, K. P. Batts, L. J. Burgart et al., "Early detection of hepatitis $\mathrm{C}$ allograft reinfection after orthotopic liver transplantation: a molecular and histologic study," Modern Pathology, vol. 13, no. 3, pp. 229-237, 2000.

[3] A. Regev, E. Molina, R. Moura et al., "Reliability of histopathologic assessment for the differentiation of recurrent hepatitis C from acute rejection after liver transplantation," Liver Transplantation, vol. 10, no. 10, pp. 1233-1239, 2004.

[4] E. Osman, E. Gamal, A. Gad, E. Soliman, E. A. Mohammad, and A. Hatia, "Factors affecting recurrence of hepatitis $C$ virus in adult living donor liver transplantation among Egyptian patients," Macedonian Journal of Medical Sciences, vol. 4, no. 1, pp. 64-69, 2011.

[5] A. J. Demetris, "Evolution of hepatitis C virus in liver allografts," Liver Transplantation, vol. 15, Supplement S2, pp. S35-S41, 2009.

[6] B. Roche and D. Samuel, "Risk factors for hepatitis C recurrence after liver transplantation," Journal of Viral Hepatitis, vol. 14, no. s1, pp. 89-96, 2007.

[7] H. Reis, J. Wohlschläger, S. Hagemann et al., “(Cleaved) CK18 serum and tissue expression levels differentiate acute HCV reinfection from acute rejection in liver allografts," Liver International, vol. 35, no. 3, pp. 905-913, 2015.

[8] S. J. Knechtle and J. Kwun, "Unique aspects of rejection and tolerance in liver transplantation," Seminars in Liver Disease, vol. 29, no. 1, pp. 91-101, 2009.

[9] A. Shaked, R. M. Ghobrial, R. M. Merion et al., "Incidence and severity of acute cellular rejection in recipients undergoing adult living donor or deceased donor liver transplantation," American Journal of Transplantation, vol. 9, no. 2, pp. 301308, 2009.

[10] M. Schmeding, A. Dankof, V. Krenn et al., "C4d in acute rejection after liver transplantation-a valuable tool in differential diagnosis to hepatitis C recurrence," American Journal of Transplantation, vol. 6, no. 3, pp. 523-530, 2006.

[11] D. Joshi, M. Pinzani, I. Carey, and K. Agarwal, "Recurrent HCV after liver transplantation-mechanisms, assessment and therapy," Nature Reviews Gastroenterology \& Hepatology, vol. 11, no. 12, pp. 710-721, 2014.

[12] J. R. Burton Jr and H. R. Rosen, "Acute rejection in HCVinfected liver transplant recipients: the great conundrum," Liver Transplantation, vol. 12, no. S2, Supplement S2, pp. S38-S47, 2006.

[13] C. Varol, A. Mildner, and S. Jung, "Macrophages: development and tissue specialization," Annual Review of Immunology, vol. 33, no. 1, pp. 643-675, 2015.

[14] F. Heymann and F. Tacke, "Immunology in the liver - from homeostasis to disease," Nature Reviews Gastroenterology \& Hepatology, vol. 13, no. 2, pp. 88-110, 2016.

[15] Z. X. Liu, S. Govindarajan, and N. Kaplowitz, "Innate immune system plays a critical role in determining the progression and severity of acetaminophen hepatotoxicity," Gastroenterology, vol. 127, no. 6, pp. 1760-1774, 2004.

[16] J. A. Harris, S. Jain, Q. Ren, A. Zarineh, C. Liu, and S. Ibrahim, "CD163 versus CD68 in tumor associated macrophages of classical Hodgkin lymphoma," Diagnostic Pathology, vol. 7, no. 1, pp. 12-19, 2012.

[17] Z.-F. Yang, D. W.-Y. Ho, A. C.-Y. Chu, Y.-Q. Wang, and S.-T. Fan, "Linking inflammation to acute rejection in smallfor-size liver allografts: the potential role of early macrophage activation," American Journal of Transplantation, vol. 4, no. 2, pp. 196-209, 2004.

[18] P. H. McGuinness, D. Painter, S. Davies, and G. W. McCaughan, "Increases in intrahepatic CD68 positive cells, MAC387 positive cells, and proinflammatory cytokines (particularly interleukin 18) in chronic hepatitis C infection," Gut, vol. 46, no. 2, pp. 260-269, 2000.

[19] J. Brownell and S. J. Polyak, "Molecular pathways: hepatitis C virus, CXCL10, and the inflammatory road to liver cancer," Clinical Cancer Research, vol. 19, no. 6, pp. 1347-1352, 2013.

[20] B. Li, W. Xu, L. Xu et al., "I-TAC is a dominant chemokine in controlling skin intragraft inflammation via recruiting 
$\mathrm{CXCR}^{+}$cells into the graft," Cellular Immunology, vol. 260, no. 2, pp. 83-91, 2010.

[21] D. Sirena, B. Lilienfeld, M. Eisenhut et al., "The human membrane cofactor CD46 is a receptor for species B adenovirus serotype 3," Journal of Virology, vol. 78, no. 9, pp. 4454-4462, 2004.

[22] A. G. Abdou, N. Y. Asaad, N. Ehsan, S. Younes, A. I. Gomaa, and W. Elgendy, "The diagnostic impact of C4d, CD68, and $\mathrm{NF}-\kappa \mathrm{B}$ expression in the differentiation between recurrent hepatitis $\mathrm{C}$ and acute cellular rejection after liver transplantation," Applied Immunohistochemistry \& Molecular Morphology, vol. 24, no. 9, pp. 639-647, 2016.

[23] Y. Lavin, D. Winter, R. Blecher-Gonen et al., “Tissue-resident macrophage enhancer landscapes are shaped by the local microenvironment," Cell, vol. 159, no. 6, pp. 1312-1326, 2014.

[24] K. R. Karlmark, R. Weiskirchen, H. W. Zimmermann et al., "Hepatic recruitment of the inflammatory $\mathrm{Grl}^{+}$monocyte subset upon liver injury promotes hepatic fibrosis," Hepatology, vol. 50, no. 1, pp. 261-274, 2009.

[25] F. Heymann, J. Peusquens, I. Ludwig-Portugall et al., "Liver inflammation abrogates immunological tolerance induced by Kupffer cells," Hepatology, vol. 62, no. 1, pp. 279-291, 2015.

[26] P. I. Terasaki and J. Cai, "Humoral theory of transplantation: further evidence," Current Opinion in Immunology, vol. 17, no. 5, pp. 541-545, 2005.

[27] C. G. Antoniades, A. Quaglia, L. S. Taams et al., "Source and characterization of hepatic macrophages in acetaminopheninduced acute liver failure in humans," Hepatology, vol. 56, no. 2, pp. 735-746, 2012.

[28] S. Epelman, K. J. Lavine, and G. J. Randolph, "Origin and functions of tissue macrophages," Immunity, vol. 41, no. 1, pp. 21-35, 2014.

[29] T. Sawada, A. Shimizu, K. Kubota, S. Fuchinoue, and S. Teraoka, "Lobular damage caused by cellular and humoral immunity in liver allograft rejection," Clinical Transplantation, vol. 19, no. 1, pp. 110-114, 2005.

[30] N. G. Sandler, C. Koh, A. Roque et al., "Host response to translocated microbial products predicts outcomes of patients with HBV or HCV infection," Gastroenterology, vol. 141, no. 4, pp. 1220-1230.e3, 2011.

[31] P. L. Zhang, S. K. Malek, T. M. Blasick et al., "C4d positivity is often associated with acute cellular rejection in renal transplant biopsies following Campath-1H (alemtuzumab) induction," Annals of Clinical \& Laboratory Science, vol. 37, no. 2, pp. 121-126, 2007.

[32] T. Ohtsuki, K. Kimura, Y. Tokunaga et al., "M2 macrophages play critical roles in progression of inflammatory liver disease in hepatitis C virus transgenic mice," Journal of Virology, vol. 90, no. 1, pp. 300-307, 2015.

[33] Z. Wu, M. Han, T. Chen, W. Yan, and Q. Ning, "Acute liver failure: mechanisms of immune-mediated liver injury," Liver International, vol. 30, no. 6, pp. 782-794, 2010.

[34] A. Bendelac, P. B. Savage, and L. Teyton, "The biology of NKT cells," Annual Review of Immunology, vol. 25, no. 1, pp. 297-336, 2007.

[35] D. Rossi and A. Zlotnik, "The biology of chemokines and their receptors," Annual Review of Immunology, vol. 18, no. 1, pp. 217-242, 2000.

[36] D. Jiang, J. Liang, J. Hodge et al., "Regulation of pulmonary fibrosis by chemokine receptor CXCR3," The Journal of Clinical Investigation, vol. 114, no. 2, pp. 291-299, 2004.
[37] C. H. Kim, B. Johnston, and E. C. Butcher, "Trafficking machinery of NKT cells: shared and differential chemokine receptor expression among $\mathrm{V} \alpha 24^{+} \mathrm{V} \beta 11^{+} \mathrm{NKT}$ cell subsets with distinct cytokine-producing capacity," Blood, vol. 100, no. 1, pp. 11-16, 2002.

[38] S. Lacotte, S. Brun, S. Muller, and H. Dumortier, "CXCR3, inflammation, and autoimmune diseases," Annals of the New York Academy of Sciences, vol. 1173, no. 1, pp. 310-317, 2009.

[39] A. Bonacchi, P. Romagnani, R. G. Romanelli et al., "Signal transduction by the chemokine receptor CXCR3: activation of Ras/ERK, Src, and phosphatidylinositol 3-kinase/Akt controls cell migration and proliferation in human vascular pericytes," Journal of Biological Chemistry, vol. 276, no. 13, pp. 9945-9954, 2001.

[40] B. Johnston, C. H. Kim, D. Soler, M. Emoto, and E. C. Butcher, "Differential chemokine responses and homing patterns of murine TCR $\alpha \beta$ NKT cell subsets," The Journal of Immunology, vol. 171, no. 6, pp. 2960-2969, 2003.

[41] S. M. Curbishley, B. Eksteen, R. P. Gladue, P. Lalor, and D. H. Adams, "CXCR3 activation promotes lymphocyte transendothelial migration across human hepatic endothelium under fluid flow," The American Journal of Pathology, vol. 167, no. 3, pp. 887-899, 2005. 


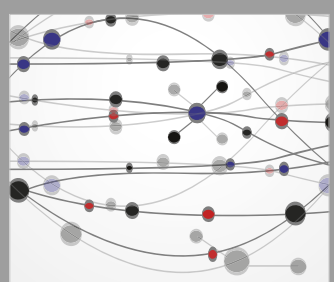

The Scientific World Journal
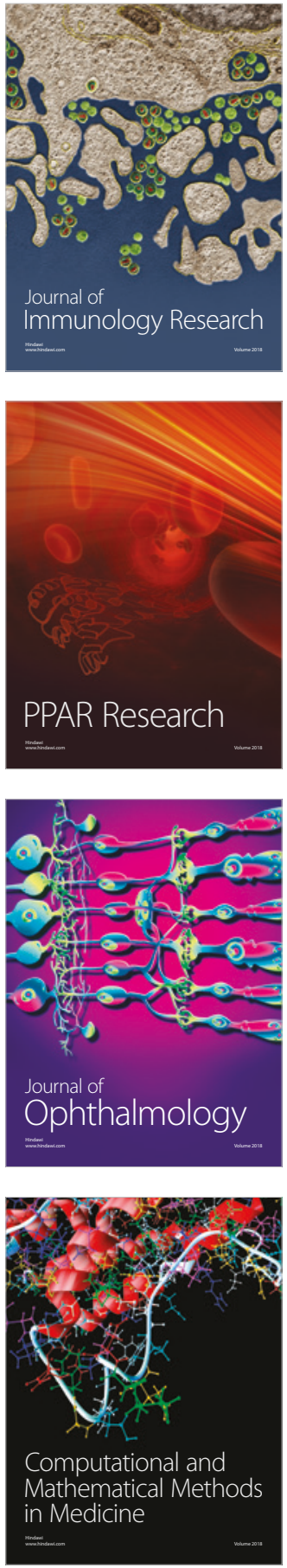

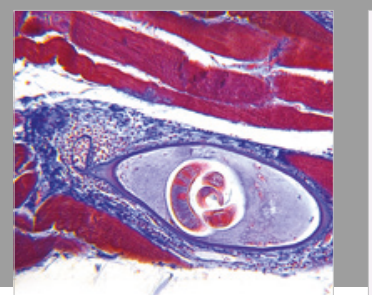

Gastroenterology Research and Practice

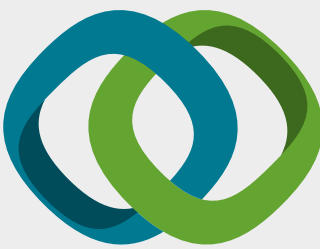

\section{Hindawi}

Submit your manuscripts at

www.hindawi.com
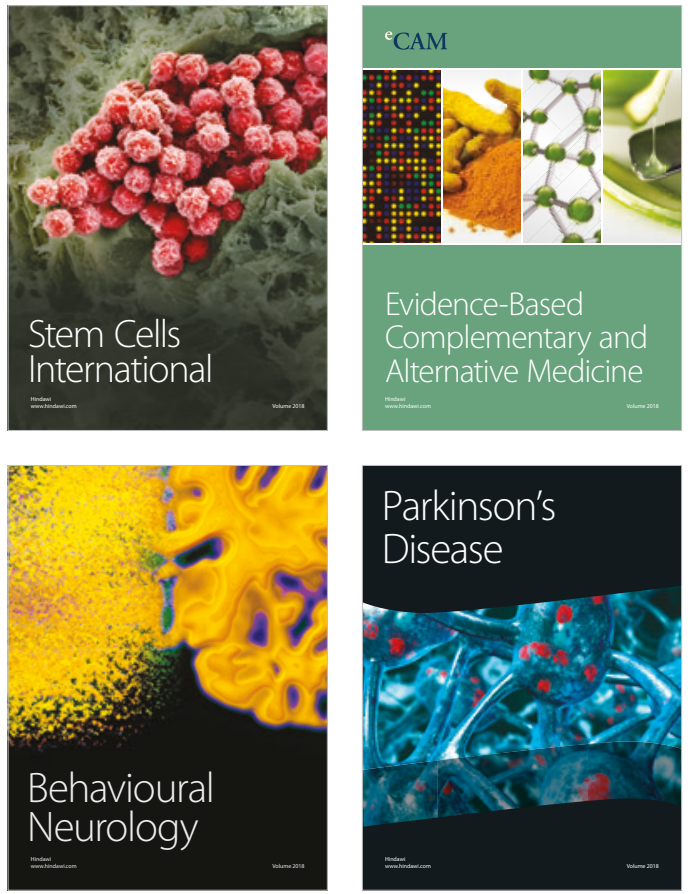

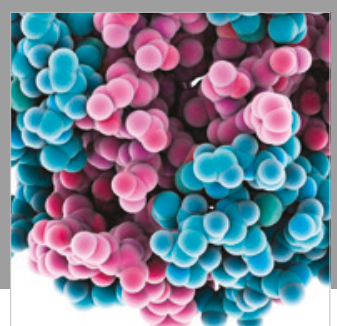

ournal of

Diabetes Research

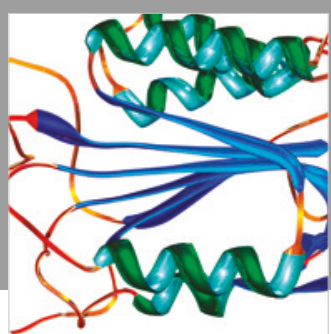

Disease Markers
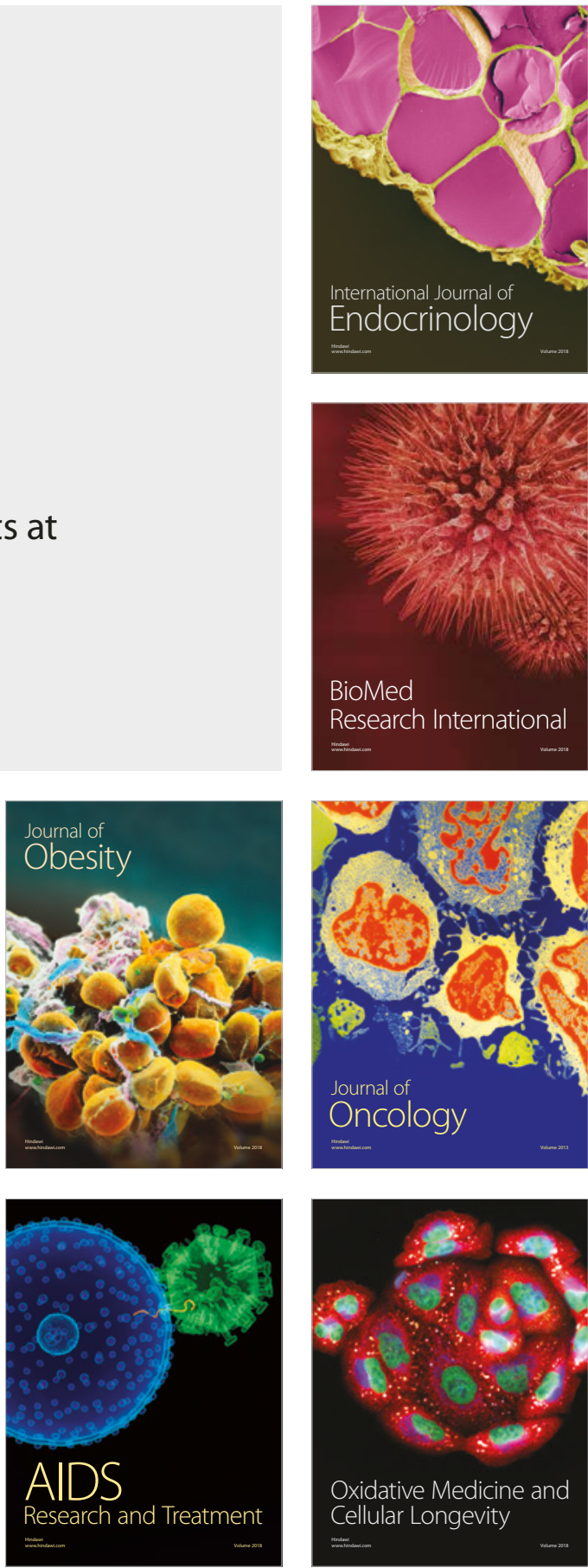